\title{
Determination of the Reliability Interval of Outdoor Channel Model in 802.15.4/ZigBee Networks
}

\author{
Héctor Kaschel $^{1}$, Gustavo Quezada ${ }^{1}$, Ricardo Vega ${ }^{2}$, Luis Sanchez ${ }^{3}$, José Mardones ${ }^{1}$ \\ 1 Departamento de Ingeniería Eléctrica, \\ Universidad de Santiago de Chile, \\ Av. Ecuador 3519, Estación Central, Santiago, Chile, \\ \{hector.kaschel; gustavo.quezada; jose.mardones\}@usach.cl \\ 2 Departamento de Ingeniería Química, \\ Universidad de Santiago de Chile, \\ Av. Ecuador 3519, Estación Central, Santiago, Chile, \\ ricardo.vega@usach.cl \\ 3 Departamento de Matemáticas y Ciencias de la Computación, \\ Universidad de Santiago de Chile, \\ Av. Ecuador 3519, Estación Central, Santiago, Chile, \\ luis.sanchez@usach.cl
}

Abstract: Within the framework of the implementation of a wireless network of nodes under the IEEE802.15.4/Zigbee standard, the values obtained from experimental measurements of power as a function of distance between nodes in free space are analyzed. A propagation loss model is proposed and a statistical analysis is made to determine the reliability of the prediction of the distance between nodes as a function of the power measured at the receiver. This analysis confirms that the predictions obtained from the proposed model and from widely used models, are not appropriate for determining, with a high degree of certainty, the distance between transmitter and receiver due to the variability of the data.

Keywords: Wireless Sensor Network, Prediction methods, Outdoor, Zigbee, Channel models, Propagation.

\section{Introduction}

In communications systems such as wireless sensors based on RF (Radio Frequency), the propagation of the waves undergoes fading due to distance and to refraction, diffraction, dispersion, and the Doppler effect if there is motion between the emitter and the receiver [1]. Fading causes a decrease of the signal's intensity at the receiver, and that can be a source of error because the receivers need a minimum signal intensity to perform an adequate demodulation [2]. There are models that allow the prediction of the intensity of the signal received in a wireless communication between the transmitter and the receiver considering the effects mentioned above [1].

This paper reports on experimental measurements with COTS (Comercial OffThe-Shelf) IEEE802.15.4 devices [2], specifically Xbee series I, for point-to-point communications with LOS (Line Of Sight). The obtained values are analyzed and from these a fitting function is proposed that represents power as a function of distance. Then a statistical analysis is applied to the proposed model, determining from that analysis its pertinence for predicting the values of the distance between nodes from the power of the receiver.

The importance of this analysis lies in the fact that the prediction of the signal's intensity in the receiver can be useful to determine the distance between two devices and their relative position [3], [4], [5], [6], [7]. From this information it is possible to establish the power required to make the connection with the following node, and it is a useful parameter for selecting routes in the case of networks with multihops [8], [9], [10], [11], [12] similar to generate selfsimilar traffic in computer network [13].

The arrangement of the paper is the following: Section II presents models of path loss. Section III presents experimental values obtained for distance loss. Section IV presents the fitting of the trend curves for the experimental values. Section V presents the analysis of the models found. Finally, Section VI presents the conclusions of the work.

\section{Models of Path Loss}

In large scale propagation models it is assumed that propagation through free space 
is subject to power loss dependent on distance, called path loss. The power received at a distance $d$ in meters between transmitter and receiver, with line of sight, is described by the free space Friis equation [1]:

$$
P_{r x}(d)=\frac{P_{t x} G_{t} G_{r} \lambda^{2}}{(4 \pi)^{2} d^{2} L}
$$

where $P_{t x}$ is the transmitting power, $P_{r x}(d)$ is the power delivered to the receiver as a function of distance $d$ between transmitter and receiver, $G_{t}$ and $G_{r}$ are the gains of the transmitting and receiving antennas, respectively, $\lambda$ is the wavelength in meters, and $L \geq 1$ summarizes the losses along the transmission lines, filter losses and antenna losses in the communication system that are not associated with the propagation itself. Table 1 shows the parameters associated with the Xbee devices, considering antennas of the chip and whip types [14]]. These parameters are used to draw the graph that shows the relation between distance and path loss applying the model presented in (1).

Table I. System's Parameters

\begin{tabular}{|c|c|c|}
\hline & $\begin{array}{c}\text { Chip Antenna } \\
\text { (experiment 1) }\end{array}$ & $\begin{array}{c}\text { Whip Antena } \\
\text { (experiment 2) }\end{array}$ \\
\hline $\mathrm{Gt}$ & $-1.5 \mathrm{dBi}$ & $1.5 \mathrm{dBi}$ \\
\hline $\mathrm{Gr}$ & $-1.5 \mathrm{dBi}$ & $1.5 \mathrm{dBi}$ \\
\hline $\begin{array}{c}\mathrm{f}_{\mathrm{c}} \text { (freq. } \\
\text { Carrier) }\end{array}$ & $2400(\mathrm{MHz})$ & $2400(\mathrm{MHz})$ \\
\hline$\lambda$ & $0.125 \mathrm{~m}$ & $0.125 \mathrm{~m}$ \\
\hline $\mathrm{L}$ & 1 & 1 \\
\hline $\mathrm{P}_{\mathrm{tx}}$ & $1 \mathrm{~mW}=0 \mathrm{dBm}$ & $1 \mathrm{~mW}=0 \mathrm{dBm}$ \\
\hline
\end{tabular}

Figure 1 shows the loss curve as a function of distance to apply the model of Friis with the values of Table 1 , simulated with the Scilab software [15].

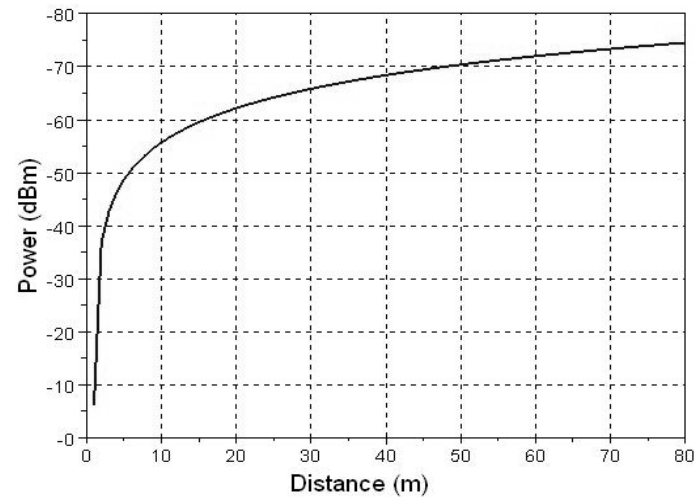

Figure 1. Theoretical loss calculated with the Friis equation
An empirical model based on path loss is presented in [2]:

$$
\begin{aligned}
P_{d}= & P_{0}-10 \cdot 2 \cdot \log _{10}(f) \\
& -10 \cdot 2 \cdot \log _{10}(d)+27.56
\end{aligned}
$$

where $P_{d}$ and $P_{0}$ represent the signal power in $d B m$ at a distance $d$ and at the antenna output, respectively, and $f$ is the carrier frequency in $\mathrm{MHz}$. Figure 2 shows the results obtained by simulating this model on the Scilab software. The dashed line represents the curve obtained for the chip antenna and the solid line for the whip antenna. Other analyses, such as the tworay model [1], are not relevant because the altitude and distance between transmitter and receiver used in WSN are relatively small.

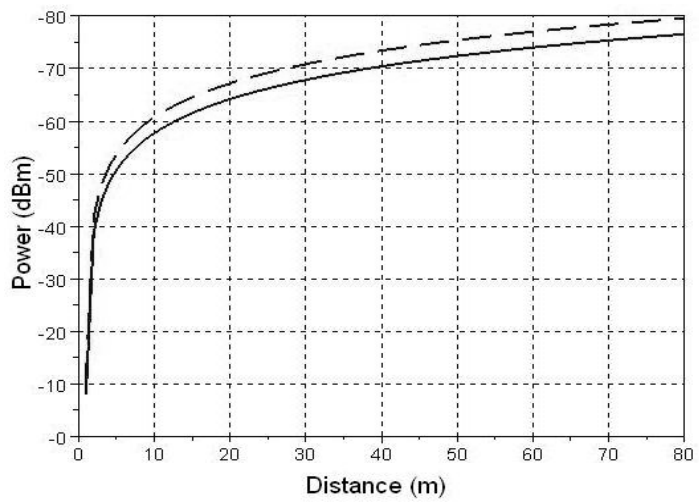

Figure 2. Theoretical loss calculated by means of the empirical model of Seydel-Rappaport using chip type (dashed line) and whip type (solid line) antennas.

\section{Experimental Path Loss Values}

Two experiments were made, using XBee series I devices in both of them. The measurements were made in an open area without obstacles on a grass covered surface. The nodes were placed at a height of $1.20 \mathrm{~m}$ and the distance between them was increased at a rate of $1(\mathrm{~m})$ per measurement, keeping the receiver fixed and displacing the transmitter.

Figure 3 shows the data frame structure at the receiver node, from which we get the power values in $\mathrm{dBm}$ contained in the RSSI (Received Signal Strength Indicator) parameter, which can be used to estimate the distance and position in WSN [16]. For the recovery of the data frame and the later identification of the RSSI parameter, an algorithm was programmed in the JAVA programming language JAVA, which recovers the desired value in real time. 


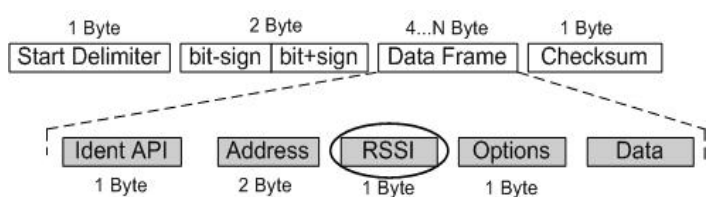

Figure 3. Mesh structure of the API mode.

In the first experiment Xbee devices with flat monopole type antennas, known as chip antennas, were used, which are placed over the transceptor devices [14]. These are also used in other devices to implement WPANs (Wireless Personal Area Network) such as Bluetooth and Wi-Fi devices [14]. Figure 4 shows the measurements obtained from the first experiment.

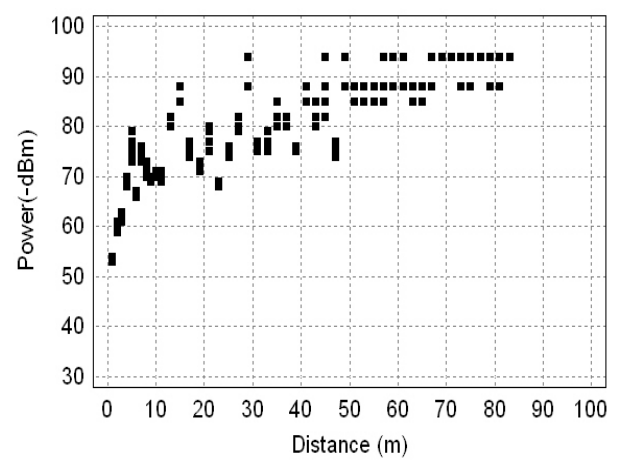

Figure 4. Experimental measurement of the free air path loss in the first experiment.

The dispersion of the data obtained can be accounted for in part by the irregular characteristics of the lobe of the chip antenna [14] used in the experiment.

Figure 5 shows the experimental data obtained in the second experiment, for the XBee devices equipped with a whip antenna, showing less data dispersion. The experimental procedure is the same as that employed for the devices with flat monopole type antennas.

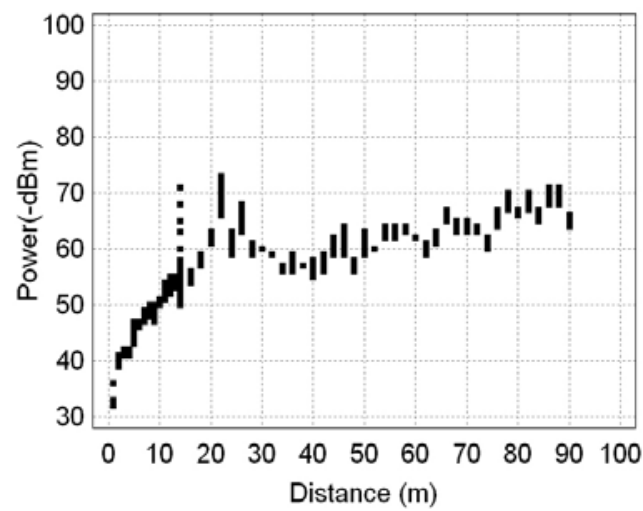

Figure 5. Experimental measurement of the free air path loss in the second experiment.

\section{Fitting of the Trend Curves}

To establish the relation between the distance and received power variables, a regression analysis was made between the variables obtained in the experimental stage presented above. The main idea of this analysis is to establish a prediction model using the experimental values. To find the adequate fitting function a heuristic search was made using the Statgraphics and Curveexpert statistical analysis software [17], [18]. The parameter applied for selecting the fitting function was the correlation coefficient [18].

The selected function is the following:

$y=a x^{b}$

where $x$ represents the independent variable "distance" in meters, and $y$ represents the dependent variable "power" in $\mathrm{dBm}$. Constants $a$ and $b$ represent the best fit of the curve to the measurements, and they are obtained by applying mean least squares. The resultant correlation coefficients were 0.8869 and 0.9293 for the first and the second experiments, respectively. The best fit values of the curve are shown in equations (4) and (5) for the first and the second experiments, respectively.

$$
\begin{aligned}
& y=52.883 \cdot x^{0.1283} \\
& y=36.733 \cdot x^{0.1355}
\end{aligned}
$$

The next step is to test the significance of the regression, for this which use is made of the ratio between the mean square of the regression and the mean square residual referred to the $F_{1, n-2}$ distribution [19]. Calculating this ratio yields an experimental value for $F$ equal to 5372, indicating that the regression is highly significant, with a confidence level much better than 95\% [19]. Figure 6 shows the fitted curve of the measurements made in experiment 1. 


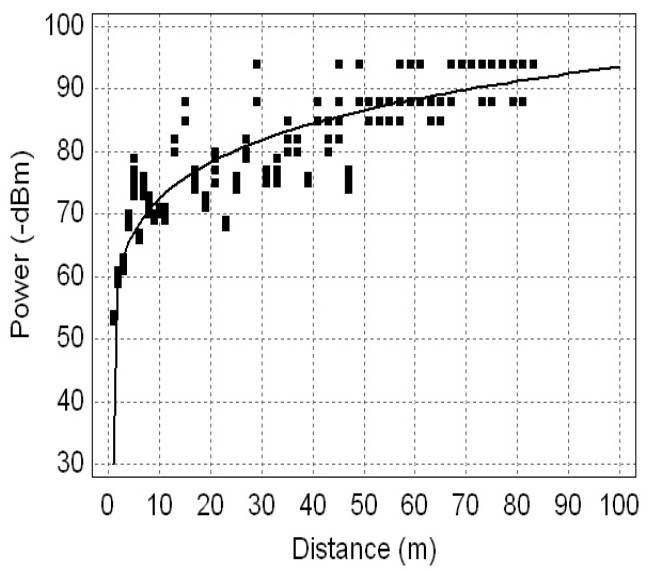

Figure 6. Fitted curve for experiment 1.

Figure 7 shows the fitted curve of the measurements made in experiment 2.

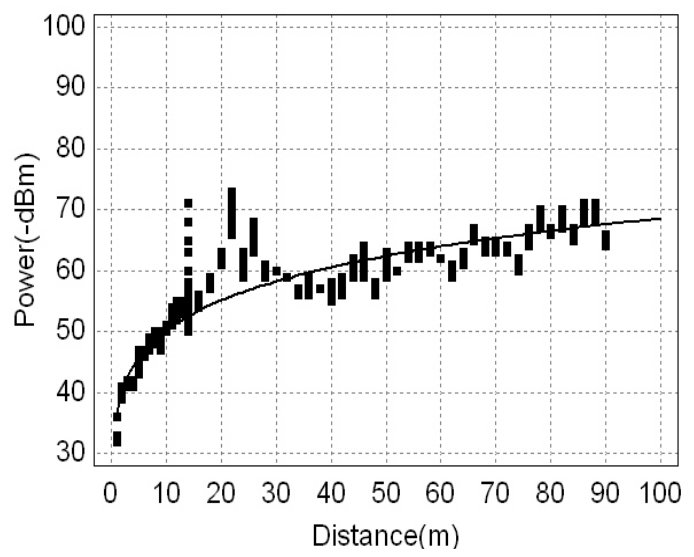

Figure 7. Experimental measurement of the free air path loss, with the fitted curve, for experiment 2.

Figure 8 presents the curves of the theoretical models of Seidel-Rappaport and Friis shown as solid lines, together with the curves obtained from the first and second experiments present in the third section, as dashed lines. The upper dashed curve represents the first experiment, with a worse performance than the theoretical models. The lower dashed line represents the second experiment, with better performance than the theoretical models.

This behavior indicates that particular characteristics of each device lead to better or worse performance with respect to the theoretical models, which would indicate a "mean" performance value.

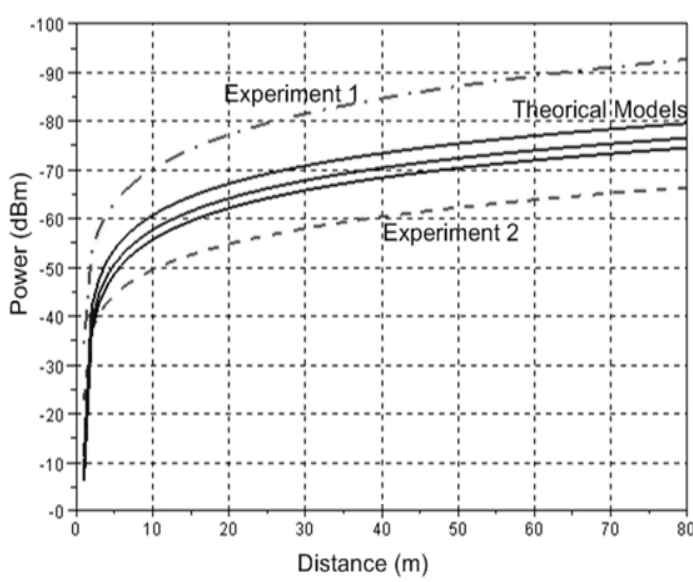

Figure 8. Comparison of the curves. The dashed lines correspond to the experimental fit, and the solid lines correspond to those obtained with the models of Seidel-Rappaport and Friis.

\section{Analysis of the Experimental Models}

To evaluate the proposed models in this paper, the experimental data were analyzed with the Statgraphics statistical software [17]. It is seen that the $p$-value obtained from the analysis of variance (ANOVA) [18] is lesss than 0.01 , which indicates a statistically significant relation between the distance and the power for a confidence level of $95 \%$ for both experiments.

For the first and second experiments, Rsquared indicates that the model explains $78.6584 \%$ and $86.3682 \%$ of the power variation, respectively. Therefore the regression models may not provide precise predictions of future observations. The standard error of the estimation shows that the typical deviation of the residuals is 0.0787 and 0.0579 for the first and second experiments, respectively, values which are not usable to construct prediction limits for new observations.

The residuals graphs, Figures 9 and 10, present the studentized residuals of all the observations and indicate the typical deviation of each observed value of $y$ (power) with respect to the fitting model for each experiment [19]. Atypical ones are those greater than 2.0 as absolute value. In this case the values are not many and they indicate that the model adequate itself to the measured values in both experiments. 


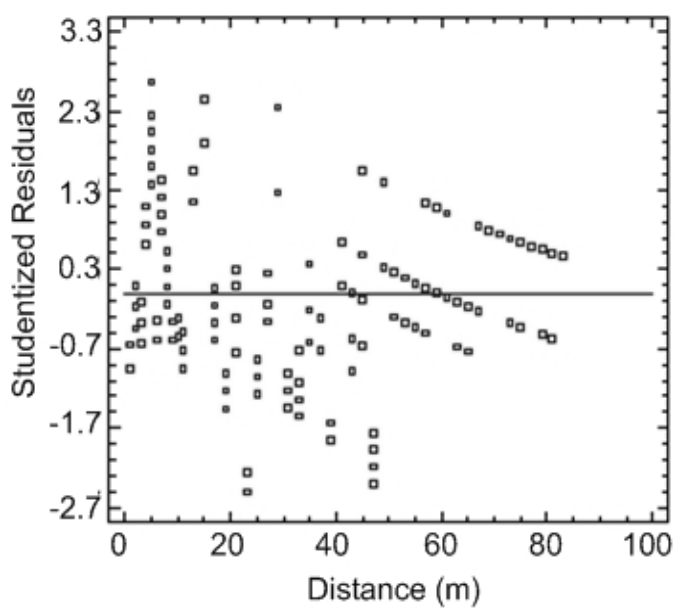

Figure 9. Graph of residuals associated with observations of experiment 1.

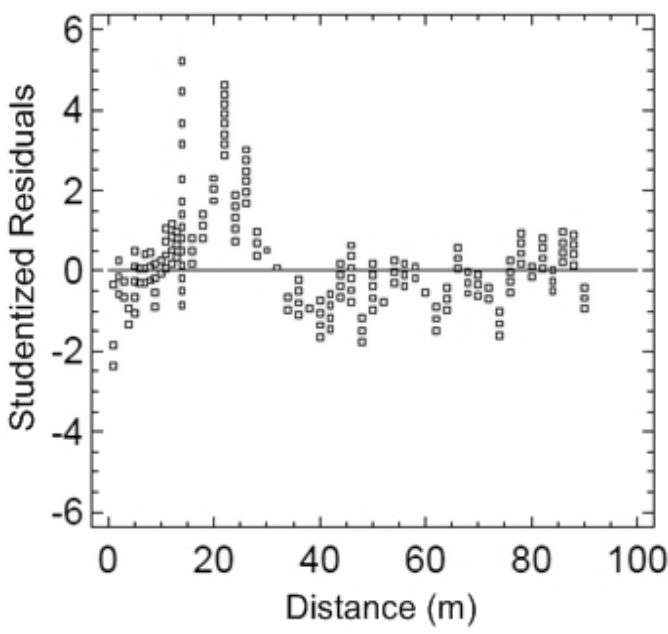

Figure 10. Graph of residuals associated with observations of experiment 2

Finally, the lack of fit test [19] was applied to the linearized curves to determine whether the chosen model is adequate to describe the observed data. The test is made comparing the variability of the current model's residuals with the variability of the observations of the values of the independent variable $x$ (distance). Since the p-value for the lack of fit obtained from ANOVA is less than 0.01, there is a statistically significant lack of fit for a confidence level of $99 \%$, which implies that the models are not the most appropriate, in view of the variability of the data, for both experiments.

\section{Conclusions}

This work determines loss as a function of distance from models widely used in wireless communications for open spaces. Then experimental measurements are made and an empirical model is proposed for predicting loss by means of a regression analysis of the data. The proposed model is compared with known models, and finally the degree of statistical validity of the model found is analyzed.

The models presented in the literature and the model proposed from the experimental analysis are very similar. However, these models are not appropriate to determine with a high degree of certainty the distance between the transmitter and the receiver from the power because of the variability of the data. This variability may be due to the low power levels used as detection threshold in the receivers, and on the other hand because of the irregular coverage lobes of the transceptor's antennas, particularly the chip type antennas. Even though the use of devices with external whip type antennas increases the coverage and decreases the variability of the data, it is not sufficient to allow the determination with a high degree of certainty the distance between the devices as a function of their power. Therefore, it is suggested to use other techniques or hardware if it is desired to achieve accuracy in predicting the distance between devices.

The analysis is made for an open space scenario with line of sight. It can therefore be foreseen that in confined spaces and with obstructions these types of models present an even worse performance for determining the distance as a function of the received power.

Future work may consider systems with more power and antennas of the directional type to achieve models with better performance. From the results obtained in this work is working on additional methods to achieve greater statistical certainty.

\section{Acknowledgements}

Financial support under DICYT USACH projects Code 060913KC "Design and implementation of a fault tolerant IWSN (Industrial Wireless Sensor Network) with Cooperative Diversity" and Code 060933SYB "Development of a layer architecture to distribute functions to sensors and actuators, under reliability and communication criteria, in an industrial network of wireless sensors". 


\section{REFERENCES}

1. RAPPAPORT, T. S., Wireless Communications - Principles and Practice. Prentice Hall, Upper Saddle River, NJ, 2002.

2. HOLGER, K., A. WILlig, Protocols and Architectures for Wireless Sensor Network, John Wiley and Sons, 2005.

3. HOOD, B. N., P. BAROOAH, Estimating DoA from RadioFrequency RSSI Measurements Using an Actuated Reflector, IEEE Sensors Journal, vol. 11, Feb. 2011.

4. LEE, K., B. KIM, H. LEE, Y. SHIN, Improving Localization Accuracy Using Signal Attenuation due to Neighbour Signal Interference in Wireless Sensor Networks. $13^{\text {th }}$ International Conference on Advanced Communication Technology (ICACT), 13-16 Feb. 2011.

5. LAU, E.-E.-L., B.-G. LEE, S.-C. LEE, W.-Y. CHUNG, Enhanced RSSI-based High Accuracy Real-time User Location Tracking System for Indoor and Outdoor Environments. International Journal on Smart Sensing and Intelligent Systems, vol. 1, no. 2, June 2008.

6. LI, J., H.-P. LIU, A New Weighted Centroid Localization Algorithm in Coal Mine Wireless Sensor Networks, 3rd International Conference on Computer Research and Development (ICCRD), 11-13 March 2011.

7. CASEY, P. R., K. E. TEPE, N. KAR, Design and Implementation of a Testbed for IEEE 802.15.4 (Zigbee) Performance Measurements. EURASIP Journal on Wireless Communications and Networking Volume 2010, ID 103406, 2010.

8. BARSOCCHI, P., S. LENZI, S. CHESSA, member IEEE, G. GIUNTA, Pisa Research Area, Via G.Moruzzi. Virtual Calibration for RSSI-based Indoor Localization with IEEE 802.15.4. ICC'09. IEEE International Conference on Communications, 2009.
9. PRADIP, D., Y. LIU, S. K. DAS, Energy-Efficient Reprogramming of a Swarm of Mobile Sensors. IEEE Transactions on Mobile Computing, May 2010.

10. QUEZADA, G., J. MARDONES, A. SOTO, Applied Research Line to Industrial Wireless Sensor Networks. Valdivia, Chile. August 2008. INGELECTRA 2008.

11. KASCHEL, H., G. QUEZADA, A. SOTO, J. MARDONES, Requirements to Industrial Wireless Sensor Networks under Cooperative Diversity Operation. XVIII Congreso Asociación Chilena de Control Automático. Santiago Chile 10-12, December 2009, pp. 1-6.

12. VURAL, S., E. EKICI, On Multihop Distances in Wireless Sensor Networks with Random Node Locations, IEEE Transactions on Mobile Computing, vol. 9, no. 4, April 2010.

13. MILLÁN, G., H. KASCHEL, G. LEFRANC, A Simple Model for the Generation of LRD Self-similar Traffic Using Piecewise Affine Chaotic Onedimensional Maps, in SIC, vol. 19, No. 1, 2010, pp. 67-78

14. User manual Fractus Reach Xtend ${ }^{\mathrm{TM}}$ Bluetooth ${ }^{\circledR}$, 802.11b/g WLAN Chip Antenna. Abril 2008.

15. http://www.scilab.org/

16. RUKPAKAVONG, W., I. PHILLIPS, G. LIN, Neighbour Discovery for Transmit Power Adjustment in IEEE 802.15.4 Using RSSI. 4th IFIP International Conference on New Technologies, Mobility and Security (NTMS), Feb. 2011.

17. http://www.statgraphics.com/

18. http://curveexpert.webhop.net/

19. MONTGOMERY, D. C., RUNGER G. C., Applied Statistics and Probability for Engineers, 3rd. Ed., John Wiley \& Sons, USA, 2003 